\section{The Overview of the Management of Comorbid Cardiovascular Diseases and Diabetes during COVID-19}

Abstract
Coronaviruses are positive singlestranded RNA viruses which are spread and transmitted to both humans and animals all around the world. The virus that causes COVID-19 disease is in a family of viruses that is called Coronaviridae. Most people infected by this virus will experience mild to moderate respiratory problems. Older people and those with underlying medical problems such as cardiovascular disease, diabetes, chronic respiratory disease, and cancer are more likely to develop serious illness and this virus is more dangerous to these patients. Until now there is no exact treatment for this disease. During the pandemic, one of the challenging parts for better clinical outcomes is the management of patients with comorbid cardiovascular diseases. Patients with previous or underlying cardiovascular diseases are at higher risk for developing severe symptoms if infected with SARS$\mathrm{CoV}-2$. Several mechanisms can lead to cardiovascular complications in patients with COVID-19 such as the direct viral injury to complications, which is secondary to the inflammatory and thrombotic responses to the infection. SARS-CoV-2 infection downregulates angiotensin-converting enzyme 2 (ACE2) expression, which might have an important execution in COVID-19. It is a therapeutic abeyant which is becoming very attractive for the cure and prevention of COVID-19. Further research is needed to continue or discontinue ACE inhibitors and angiotensin receptor blockers, which are chief in hypertension and heart failure in COVID-19. Diabetes is another widely spread comorbid disease, and this is one of the main causes of morbidity and mortality all over the world during 


\begin{abstract}
COVID-19. Infection of SARS-COV-2 in the patients who have diabetes activate higher stress conditions, and this leads to the greater release of hyperglycemic hormones, e.g., glucocorticoids and catecholamines. This paper emphasizes on COVID-19 and comorbid cardiovascular diseases with a literature review of various relevant articles, recent studies, and selected references about concepts of underlying mechanisms of pathogenesis and treatment for comorbid conditions during COVID-19.
\end{abstract}

Subject: Biology

Keywords: $\quad$ Coronavirus,Covid-19, Cardiovascular Diseases (Cvd), Diabetes, Comorbid Diseases

\section{Materials and Methods}

A literature search of international databases (PubMed, Hinari and Cochrane library, Google scholar etc) was undertaken to provide a comprehensive overview of existing research `evidence about the interactions between COVID-19 disease and comorbid diseases such as CVD, diabetes, and neurological manifestations. The search strategy involved the primary keyword "coronavirus" and "COVID-19" combined with "disease", "comorbid diseases", "Cardiovascular diseases”, "Diabetes”, and/or "neurological manifestations". Only papers written in English were included, and publication date was not restricted. During the study, the dates of papers (which had the most accurate and updated information possible) were inspected and its trustworthiness was also ensured through the referred databases. PubMed database and Google Scholar were searched using the key terms 'COVID-19', diabetes', and cardiovascular disease' up to September, 2020. Full texts of the retrieved articles were accessed.

Few data base was also given from the latest articles available on the different famous scientific American and European journals. All relevant articles providing information on relations of COVID-19 and it's comorbidities with clinical characteristics and epidemiological information was selected for review and analysis.

\section{Introduction}

In December 2019, a new kind of disease known as COVID-19 was recognized which originated from Wuhan, China (Wang et al., 2020). In 2020, 
corona virus spread worldwide and its fatality rate increased. This disease can be caused by the new strain of the coronavirus.

COVID is the abbreviation format of CO that refers to Corona, VI refers to the virus, and D refers to the disease. COVID-19 can have different symptoms from mild to severe in different patients with different immune systems. It can also lead to death especially in people with weak immune systems and background of other systemic diseases. Corona virus mostly affects the respiratory tract and can cause the following symptoms: fever, sore throat, dry cough, and struggling for breath. In severe cases, symptoms such as pneumonia, severe acute respiratory syndrome, and fatal outcome was observed. Symptoms of COVID-19 can be observed at any time, especially from 2 to 14 days after exposure and the incubation is about 2 to 5 days. There are many results which show that comorbidities increase the chance of infection. Comorbidity can be defined as the existence of more than two conditions that are co-occurring with the primary condition. Thus, there can be a correlation between COVID-19 and comorbid cardiovascular diseases. Patients with cardiovascular diseases and diabetes are faced with a higher chance of severe complications from COVID-19. Another factor that is totally important for clinical manifestations and outcome is the patient's age. This is because older patients are also at higher risks. According to recent researches and databases, patients with diabetes who have corona virus disease are at higher risk of mortality because of many factors such as the impaired immune response, especially inappropriate $\mathrm{T}$ cell action and also impaired natural killer cell activity. For patients with diabetes, the role of taking drugs such as metformin, Anti-hyperglycemic, and angiotensin receptor blocking is considerable. The reason why diabetes can increase the severity of COVID19 is unclear. However, as mentioned earlier, many factors can play a significant role in it. In reviewing these concepts, this paper will focus on comorbidity such as cardiovascular diseases and diabetes, the actual aspects, and the current recommendations for treatment.

\section{Overview}

Recent research on the COVID-19 pandemic has shown that the virus can cause problems in the cardiovascular system such as myocardial injury, heart failure, Takotsubo syndrome (TS), arrhythmias, myocarditis, and shock (Driggin et al., 2020; Hungde Hu et al., 2020; Shi et al., 2020). The cardiovascular damage duo to COVID-19 is multifactorial and may be caused by an imbalance between high metabolic demand and low cardiac reserve, systemic inflammation, thrombogenesis, and direct cardiac damage from the virus (Xiong et al., 2020). This damage to the cardiovascular system occurs mainly in patients with cardiovascular risk factors (advanced age, hypertension, and diabetes) or pre-existing CVD (Tao Gua et al., 2020; Shi et 
al., 2020). Patients with pre-existing CVD appear to have heightened vulnerability to develop COVID-19 and tend to have more severe disease with worse clinical outcomes (Bo Li et al., 2020; Huang et al., 2020; Wu et al., 2020; Wang et al., 2020).

The common mechanisms answerable for CV complications in COVID-19 are as follows: (Li et al., 2020; Xiong et al., 2020).

1. Direct myocardial injury-SARS-CoV-2 enters human cells by binding to angiotensin-converting enzyme 2 (ACE2), a membrane-bound aminopeptidase that is extremely expressed in the heart and lungs. ACE2 plays a crucial role in neurohumoral regulation of the CV system in normal health as well as in various disease conditions. The bounden of SARS-CoV-2 to ACE2 can result in changes in ACE2, which signals pathways leading to acute lung and myocardial injury (Hongde Hu et al., 2020).

2. Systemic Inflammation: The more severe style of COVID-19 is characterized by acute systemic inflammatory response and cytokine storm, which may end in injury to multiple organs resulting in multiorgan failure. Studies have shown high circulatory levels of proinflammatory cytokines in patients with severe/critical COVID-19 (Huang et al., 2020; Zhou et al., 2020).

3.Altered Myocardial Demand-Supply Ratio: Increased cardiometabolic demand related to the systemic infection coupled with hypoxia caused by acute respiratory diseases can impair myocardial oxygen demand-supply relationship and lead to acute myocardial injury (Bansal, 2020).

4.Plaque Rupture and Coronary Thrombosis: Systemic inflammation leads to increased shear stress because increased coronary blood flow can precipitate plaque rupture resulting in acute myocardial infarction. More so, the prothrombotic milieu created by systemic inflammation further increases the risk (Bansal, 2020).

5.Adverse Effects of Various Therapies: Various antiviral drugs, corticosteroids, and other therapies aimed towards treating COVID-19 can also have deleterious effects on the CV system (Bansal, 2020).

6.Electrolyte Imbalances: Electrolyte imbalances can occur in any critical systemic illness and precipitate arrhythmias, especially in patients with the underlying cardiac disorder. There is particular concern about hypokalemia in COVID-19 due to the interaction of SARS-CoV-2 with the renin-angiotensin-aldosterone system (Chen et al., 2019). Hypokalemia increases vulnerability to numerous tachyarrhythmias (Bansal, 2020).

The clinical cardiovascular manifestations of COVID-19 include cardiac symptoms such as palpitations and chest tightness (Hulot, 2020). This is with an elevation of cardiac biomarkers (ischaemic or non-ischaemic etiology), cardiac arrhythmia, arterial and venous thromboembolism (VTE), and cardiogenic shock and arrest (Kang et al., 2020). 
Studies on patients with COVID-19 have generally defined myocardial injury as the elevation of high-sensitivity cardiac troponin (hs-cTn) above the 99th percentile of its upper limit of normal or evidence of new electrocardiographic or echocardiographic abnormalities (Huang et al., 2020; Zhou et al., 2020). It also includes increased levels of hs-cTn which correlates with disease severity and mortality rate in COVID-19, even after controlling other comorbidities (Zheng et al., 2020). Severe viral infections can cause a systemic inflammatory response syndrome that increases the risk of plaque rupture and thrombus formation, resulting in either an ST-elevation MI or nonST-elevation MI (Warren-Gash et al., 2012). Severe respiratory viral infections can also lead to decreased oxygen delivery to the myocardium via hypoxemia and vasoconstriction, as well as the hemodynamic effects of sepsis with increased myocardial oxygen demand. This supply and demand imbalance may lead to sustained myocardial ischemia in patients with underlying coronary artery disease (Kang et al., 2020). Altogether, the diagnosis of acute MI should be done based on the patient's clinical symptoms, laboratory tests and findings of ECG, echocardiography, and angiography.

However, there are findings that show the correlation between COVID-19 and Myocardial injuries with DIC: Disseminated intravascular coagulation (DIC). Myocardial injury with DIC has been recently reported in two critically ill patients with COVID-19 (Wang et al., 2020). Both patients had significantly elevated Tn and brain natriuretic peptide, which normalized after treatment with heparin, mechanical ventilation, and antiviral agents (Kang et al., 2020).

A study of 137 patients in Wuhan showed that $7.3 \%$ had experienced palpitations as one of their presenting symptoms for COVID-19 (Liu et al., 2020). Arrhythmias in both forms of the bradycardia and tachycardia are known to happen in COVID-19. Arrhythmias were found to be more common in the intensive care unit (ICU) patients with COVID-19 (44.4\%) than nonICU patients (6.9\%) (Wang et al., 2020). Patients with elevated Tn also had a higher incidence of malignant arrhythmia (hemodynamically unstable ventricular tachycardia or ventricular fibrillation) than those with normal Tn levels (11.5\% vs 5.2\%, $\mathrm{p}<0.001)$ (Guo et al., 2020).

Patients with COVID-19 due to prolonged immobilization, high coagulation status, and active inflammatory responses are prone to venous thromboembolism (VTE). The prevalence of ultrasound which confirmed deep venous thrombosis in patients with COVID-19 is 22.7\% (Shi et al., 2020), while that of ICU patients is $27 \%$ (Klok et al., 2020). Patients with COVID-19 have been shown to have a significantly higher level of D-dimer, fibrin degradation products (FDP), and fibrinogen compared with healthy controls which also increased with the severity of COVID-19 (Tang et al., 
2020). Consequently, in ill COVID-19 patients, VTE and pulmonary embolism should be considered.

Diabetes is related to an increased risk of severe bacterial (Hodgso et al., 2015) and viral respiratory tract infections (Allard et al., 2010). Analysis of about 500 subjects hospitalized with SARS-CoV in China revealed that elevations in fasting glucose were associated with increased rates of death. However, hyperglycemia was often transient and is generally resolved after discharge from the hospital within the majority of subjects (Yang et al., 2010). A diagnosis of diabetes was associated with a 3-fold increased risk of mortality in the analysis of 114 adults hospitalized with SARS-CoV in Toronto in 2003 (Booth et al., 2003).

Thus, why are patients with diabetes considered 'high-risk groups'? As discussed above, human pathogenic coronaviruses bind to host target cells through angiotensin-converting enzyme 2 (ACE2), which is widely expressed by bronchial mucosal epithelial cells (Wan, et al., 2020). ACE2 expression is increased in diabetic patients prescribed ACE inhibitors and angiotensin II type-1 receptor blockers (ARBs) (Wan et al., 2020). ACE2 expression is additionally increased by ibuprofen and thiazolidinediones. Hence, the WHO has recommended that patients suffering COVID-19 symptoms should avoid taking ibuprofen. However, there is no consensus statement or scientific evidence suggesting that thiazolidinediones should be discontinued in patients with diabetes showing COVID-19 symptoms. These data suggest ACE2 expression is increased in patients with diabetes treated with ACE inhibitors and ARBs, which can facilitate infection with COVID-19. However, on the contrary, there is evidence from animal studies suggesting that ACE inhibitors and ARBs are protective against serious lung damage in COVID-19 infection, but this has not been replicated in human subjects. Therefore, due to a lack of evidence based on the harmful effects of ACE inhibitors and ARBs in the context of the pandemic COVID-19 outbreak, the Council of Hypertension of the European Society of Cardiology (ESC) released a position statement recommending that ACE inhibitors and ARBs should not be discontinued for those infected with COVID-19 (Hodgson et al., 2015).

The available evidence implicates diabetes and obesity as important risk factors impacting the clinical severity of coronavirus infections, including SARS-CoV-2. Although ACE2 and DPP4 are important physiological regulators of glucose homeostasis, there is little compelling clinical evidence that drugs targeting ACE2- or DPP4-related pathways produce differential harm or benefit within the context of human coronavirus infections. Soluble decoy receptors for ACE2 or antisera directed against ACE2 is also promising investigational interventions to dam cellular coronavirus entry.

The available information doesn't indicate increased susceptibility to coronavirus infections in children or adults with T1D. People with T1D may 
find that interruption of normal daily activities, changes in type and frequency of exercise, and alteration of diet routines may alter glucose control necessitating a reexamination of insulin requirements. An increase in blood sugar or marked change in 24-hour glucose patterns could also be an early harbinger of impending clinical infection in some individuals, prompting more frequent blood sugar and ketone monitoring. In some areas, disruption to medication supply chains may require additional vigilance and more regular communication between pharmacies, insurance companies, health care providers, and individual patients. The utilization of medicines such as acetaminophen may introduce error into some technologies used for glucometer readings or continuous glucose monitoring systems (Basu et al., 2016). Despite limitations of access to health care providers, individuals with unstable kidney function or active retinopathy may have to be seen within the diabetes clinic for assessment and appropriate therapy.

However, the metabolic consequences, if any, of those investigational agents have not been carefully studied and thus require ongoing scrutiny. DPP4 inhibitors and GLP-1R agonists may exert anti-inflammatory actions in human subjects and are successfully unable to control glucose in hospitalized patients. However, there is insufficient experience with these agents to suggest they could safely replace insulin in critically ill subjects with coronavirus infection.

Hence, the extensive historical experience with the utilization of insulin, bolstered by increasing adoption of continuous glucose monitoring, supports the continuous use of insulin because of the agent of choice within the management of severely ill subjects with diabetes and coronavirus infections.

Furthermore, there is insufficient experience with diabetes and pregnancy in subjects with SARS-CoV-2 to form tailored therapeutic recommendations. As a result, modified screening guidelines for gestational diabetes are proposed within the context of SARS-CoV-2 for people with limited access to regular clinics (Yamamoto et al., 2020).

The expression of ACE2 within the exocrine and endocrine pancreas highlights the necessity for vigilance in consideration of whether pancreatic inflammation reported in some individuals with SARS-CoV-2 infection may contribute to the exacerbation or development of diabetes in a very subset of acutely ill patients. In hospitalized individuals with deteriorating renal function, the employment of SGLT2 inhibitors and exenatide should be reconsidered or discontinued, while metformin and sulfonylurea dosing might also have to be reduced or stopped (Drucker, 2020). 


\section{Treatment Strategies for COVID-19 during Comorbid Diseases ACE inhibitors and Angiotensin Receptor Blockers}

Patients with preexisting CVD have increased serum levels of ACE2, which might contribute to the more severe manifestations in that population (Xu et al., 2020; Liu et al., 2020). Individuals with hypertension would have a higher ACE2 expression secondary to the use of angiotensin-converting enzyme inhibitors (ACEI) or angiotensin II receptor blockers (ARB), which would potentially increase the susceptibility to SARS-CoV-2 infection (Driggin et al., 2020).

However, current studies on humans have some limitations: a) assessment of a small number of individuals using those drugs, and b) the advanced age of a large part of the patients assessed $\neg \neg$ - which is an important confounder, because advanced age increases the susceptibility to infection and is the major cause of poor prognosis (Vaduganathan et al., 2020). In clinical use, ACE inhibitors do not directly affect ACE2 activity. Moreover, the enzyme plays a well-known role in the recovery of ventricular function of patients with myocardial injury because it inhibits angiotensin II activity (Rice et al., 2004).

On the other hand, angiotensin II has been suggested to consider for the cardiac injury by the coronavirus, and the administration of recombinant ACE2 would normalize angiotensin II levels. Studies with recombinant ACE2 and losartan are being conducted (Vaduganathan et al., 2020). The current recommendation is that ACEI and ARB should be continued in patients based on regular use of those drugs. This is because of the clear benefit of blood pressure control and mortality decrease in those with HF. As evidenced in randomized studies, it might also be noted that the assessment of renal functions and body hemodynamics stability is necessary for deciding on the continuation or withdrawal of the drugs (Mesquita et al., 2020).

\section{Anticoagulant Therapy}

Prophylactic anticoagulants are a necessary part of the management of hospitalized COVID-19 patients due to the high risks of VTE and arterial thromboembolism. This is also as a result of prolonged immobility and high inflammatory response in the body. Thus, the optimal thrombo-prophylaxis regimen is unclear. In a retrospective study of 449 patients with severe COVID-19, 99 patients received unfractionated heparin or low molecular weight heparin for at least 7 days. No difference in overall 28-day mortality was observed. In subgroups of patients with sepsis-induced coagulopathy score $\geq 4$, or D-dimer $>$ sixfold of the upper limit of normal, the heparin group had lower mortality compared with the no-heparin group (40.0\% vs $64.2 \%$, $\mathrm{p}=0.029)(32.8 \%$ vs $52.4 \%, \mathrm{p}=0.017)$ (Tang et al., 2020). Another concern regarding thrombo-prophylaxis is the drug-drug interaction between some 
antiviral treatments (such as Ribavirin, Lopinavir, and Ritonavir) and direct oral anticoagulants. Low molecular weight heparin is likely preferred in critically ill patients with COVID-19 if anticoagulation is used (Kang et al., 2020).

\section{Hydroxychloroquine/Chloroquine and Azithromycin}

Hydroxychloroquine (an analog of chloroquine) has been demonstrated to have an anti-SARS-CoV activity in vitro (Biot et al., 2006). Hydroxychloroquine clinical safety profile is better than that of chloroquine (during long-term use) and it allows a higher daily dose (Marmor et al., 2016). In addition, it has fewer concerns regarding drug-drug interactions (Yao et al., 2020). Similar to chloroquine, hydroxychloroquine confers antiviral effects and has an additional modulating effect on activated immune cells to decrease IL-6 expression (Sahraei et al., 2019).

Azithromycin, a macrolide antibiotic that acts against Zika and Ebola viruses in vitro (Vaduganathan et al., 2020) and suppresses inflammatory processes (Sahraei et al., 2019), has been proposed as an effective adjunct to hydroxychloroquine in COVID-19 through unclear mechanisms (Yao et al., 2020). Both chloroquine and azithromycin have generally favorable safety profiles, but they are known to cause cardiovascular side effects including the prolongation of QT interval (White, 2007).

\section{Immunosuppressive Therapy}

Although corticosteroids can have a significant effect on cytokine release syndrome associated with COVID-19 and prevent its effects on the cardiopulmonary system, it is not routinely recommended for COVID-19 as for SARS and MERS because of exacerbation of the lung injury (Mehta et al., 2020; Fardet et al., 2014). Screening patients with COVID-19 using laboratory data (increasing ferritin and erythrocyte sedimentation rate, and decreasing platelets) and a proposed score for the hemophagocytic response (Hscore) (Fardet et al., 2014) for hyper inflammation may help to identify patients for whom immunosuppression might improve their survival.

IL-6 inhibitors may have a role as immunomodulators. Tocilizumab, an inhibitor of the IL-6 receptor, could improve effectively the symptoms and prevent clinical deterioration in a retrospective case series study of 21 COVID-19 patients with the severe or critical disease (Xu et al., 2020).

\section{Mechanical Cardiopulmonary Support}

In the setting of cardiogenic shock related to COVID-19, intra-aortic balloon pump (IABP) or veno-arterial ECMO should be considered (Kang et al., 2020). Extracorporeal Cardiopulmonary Resuscitation (ECMO) has been 
used successfully to manage patients with severe respiratory failure in previous coronavirus epidemics (Zochios et al., 2020).

Data from MERS epidemics showed improved survival in patients with severe ARDS supported by venovenous ECMO (Alshahrani et al., 2018). A meta-analysis of patients with isolated viral myocarditis, supported by veno arterial ECMO, showed similar survival benefits in this group (Cheng et al., 2014).

Consequently, ECMO can also provide cardiopulmonary support and end-organ function besides the administration of antivirals and antiinflammatory therapies (Cheruku et al., 2020).

\section{Summary}

At the beginning of the COVID-19 outbreak in China, more attention was focused on patients who are suffering from diabetes because of weak prognosis of those with the infection. Initial reports started with the people with Type 2 diabetes, although fresh surveys have proved that individuals with Type 1 diabetes are also at risk of severe COVID-19 infections. The reason can be multifactorial such as age, sex, ethnicity, and having comorbidities like the hypertension and cardiovascular disease or even obesity which all contribute to risk of worse outcomes. Moreover, the clinical evaluation of the cardiovascular system in COVID-19 indicates CVD such as myocardial injury, heart failure, and different arrhythmias and inflammatory processes in the heart. Hence, they are more likely to be aggressive in the case of preexisting CVD and this includes the presence of risk factors such as age, hypertension, and diabetes. Diagnosing underlying disease is crucial for distinguishing the risk factors and choosing the appropriate treatment based on patients health status for better prognosis.

\section{Conclusion}

The COVID-19 pandemic is an emerging threat to global public health. SARS-CoV-2 is associated with comorbidities that include cardiovascular disease and diabetes. COVID-19 can cause myocardial injury, heart failure, acute coronary syndrome, arrhythmias, thromboembolism, myocarditis, and shock. Therefore, people with CVD are more susceptible to sever complication of COVID-19 such as intensive care admission and even death. Accordingly, patients with CVD should continue their treatment and disease management, continuing their ACE inhibitor or ARB therapy. The available evidence implicates diabetes as an important risk factor impacting the clinical severity of SARS-CoV-2 with underlying ACE2 and DPP4 acting mechanisms as important physiological disregulators of glucose homeostasis. It is most important to avoid disruption of medication supply chains of regular 
communication between individual diabetic patients, health care providers, insurance companies, and pharmacies.

While our current understanding of COVID-19 pathogenesis is limited, a better understanding will help us develop efficacious treatment and prevention strategies for COVID-19. In addition, it will have a high impact on increasing the quality of medical care during comorbid diseases.

\section{References:}

1. Allard, R., Leclerc, P., Tremblay, C., \& Tannenbaum, T. N. (2010). Diabetes and the severity of pandemic influenza A (H1N1) infection. Diabetes care, 33(7), 1491-1493.

2. Alshahrani, MS., Sindi, A., \& Alshamsi, F. (2018). Extracorporeal membrane oxy- genation for severe Middle East respiratory syndrome coronavirus. Ann Intensive Care 2018;8:3.

3. American College of Cardiology (2020). ACC Clinical Bulletin COVID-19 Clinical Guidance For the Cardiovascular Care Team.

4. Bansal, M. (2020). Cardiovascular disease and COVID-19. Diabetes \& Metabolic Syndrome: Clinical Research \& Reviews, 14(3), 247250. retrieved from https://doi.org/10.1016/j.dsx.2020.03.013

5. Basu, A., Veettil, S., Dyer, R., Peyser, T., \& Basu, R. (2016). Direct evidence of acetaminophen interference with subcutaneous glucose sensing in humans: a pilot study. Diabetes technology \& therapeutics, 18(S2), S2-43.

6. Biot, C., Daher, W., Chavain, N., Fandeur, T., Khalife, J., \& Dive, D. (2006). Design and synthesis of hydroxyferroquine derivatives with antimalarial and antiviral activities. J Med Chem;49:2845-2849.

7. Booth, C. M., Matukas, L. M., Tomlinson, G. A., Rachlis, A. R., Rose, D. B., Dwosh, H. A., \& Ephtimios, I. E. (2003). Clinical features and short-term outcomes of 144 patients with SARS in the greater Toronto area. Jama, 289(21), 2801-2809.

8. Chen, D., Li, X., Song, Q., Hu, C., Su, F., \& Dai, J. (2020). Hypokalemia and clinical implications in patients with coronavirus disease 2019 (COVID-19) medRxiv. 2020 doi: 10.1101/2020.02.27.20028530

9. Cheng, R., Hachamovitch, R., \& Kittleson, M. (2014). Clinical outcomes in fulmi- nant myocarditis requiring extracorporeal membrane oxygenation: A weighted meta-analysis of 170 patients. J Card Fail 2014;20:400-6.

10. Cheruku, S., Dave, S., Goff, K., Park, C., Ebeling, C., Cohen, L., Styrvoky, K., Choi, C., Anand, V., \& Kershaw, C. (2020). Cardiopulmonary Resuscitation in Intensive Care Unit Patients With Coronavirus Disease 2019. Journal of Cardiothoracic and Vascular 
Anesthesia, 34(10), 2595-2603. retrieved from https://doi.org/10.1053/j.jvca.2020.06.008

11. Costa, IBSDS., Bittar, CS., Rizk, SI., Araújo Filho, AE., Santos, KAQ., Machado, TIV., Andrade, FTA., González, TB., Arévalo, ANG., Almeida, JP., Bacal, F., Oliveira, GMM., Lacerda, MVG., Barberato, SH., Chagas, ACP., Rochitte, CE., Ramires, JAF., Kalil Filho, R., \& Hajjar, LA. (2020) The Heart and COVID-19: What Cardiologists Need to Know. Retrieved October 11, 2020, retrieved from https://pubmed.ncbi.nlm.nih.gov/32401847/

12. Disseminated Intravascular Coagulation | NHLBI, NIH. (2019, October 8). NIH. retrieved from https://www.nhlbi.nih.gov/healthtopics/disseminated-intravascular-coagulation

13. Driggin, E., Madhavan, MV., Bikdeli, B., Chuich, T., Laracy, J., \& Bondi-Zoccai, G. (2020). Cardiovascular considerations for patients, health care workers, and health systems during the coronavirus disease 2019 (COVID-19) pandemic. J Am Coll Cardiol. 2020 Mar 18;pii: S0735-1097(20)34637 retrieved from https://www.scielo.br/scielo.php?script=sci_nlinks\&pid=S0066782X202000060080500004\&lng=en

14. Drucker, D. J. (2020). Coronavirus infections and type 2 diabetesshared pathways with therapeutic implications. Endocrine reviews, 41(3), bnaa011

15. Fardet, L., Galicier, L., \& Lambotte, O. (2014). Development and validation of the HScore, a score for the diagnosis of reactive hemophagocytic syndrome. Arthritis Rheumatol 2014;66:2613-20. 10.1002/art.38690

16. Gautret, P., Lagier, J-C., \& Parola, P. (2020). Hydroxychloroquine and azithromycin as a treatment of COVID-19: results of an open-label non-randomized clinical trial. Int $\mathrm{J}$ Antimicrob Agents 2020;105949:105949. 10.1016/j.ijantimicag.2020.105949

17. Guo, T., Fan, Y., \& Chen, M. (2020). Cardiovascular implications of fatal outcomes of patients with coronavirus disease 2019 (COVID-19). JAMA Cardiol 2020. 10.1001/jamacardio.2020.1017

18. Guo, T., Fan, Y., Chen, M., Wu, X., Zhang, L., \& He, T. (2020). Cardiovascular implications of fatal outcomes of patients with coronavirus disease 2019 (COVID-19). JAMA Cardiol. 2020 Mar 27. retrieved from https://www.scielo.br/scielo.php?script=sci_nlinks\&pid=S0066782X202000060080500010\&lng=en

19. Han, H., Yang, L., \& Liu, R. (2020). Prominent changes in blood coagulation of patients with SARS-CoV-2 infection. Clin Chem Lab 
Med2020. doi:10.1515/cclm-2020-0188. [Epub ahead of print: 16 Mar 2020].

20. Hodgson, K., Morris, J., Bridson, T., Govan, B., Rush, C., \& Ketheesan, N. (2015). Immunological mechanisms contributing to the double burden of diabetes and intracellular bacterial infections. Immunology, 144(2), 171-185.

21. HS, A. (n.d.) (2020). The Effect of Coronavirus Disease 2019 on Cardiovascular Diseases. Retrieved October 11, 2020, from retrieved from https://pubmed.ncbi.nlm.nih.gov/32491073/

22. Hu, H., Ma, F., Wei, X., \& Fang, Y. (2020). Coronavirus fulminant myocarditis saved with glucocorticoid and human immunoglobulin. Eur Heart J. 2020 Mar 16;pii:ehaa190. retrieved from https://www.scielo.br/scielo.php?script=sci_nlinks\&pid=S0066782X 202000060080500014\&lng=en

23. Huang, C., Wang, Y., \& Li, X. (2020). Clinical features of patients infected with 2019 novel coronavirus in Wuhan, China. Lancet2020;395:497-506. 10.1016/S0140-6736(20)30183-5

24. Hulot, J. (2020, April). COVID-19 in patients with cardiovascular diseases. Retrieved October 11, 2020, from retrieved from https://www.ncbi.nlm.nih.gov/pmc/articles/PMC7270790/

25. Hussain, A., Bhowmik, B., \& Do Vale Moreira, N. (2020). COVID-19 and diabetes: Knowledge in progress. Retrieved October 11, from retrieved from https://www.ncbi.nlm.nih.gov/pmc/articles/PMC7144611/

26. Kang, Y., Chen, T., Mui, D., Ferrari, V., Jagasia, D., Scherrer-Crosbie, M., Chen, Y., \& Han, Y. (2020). Cardiovascular manifestations and treatment considerations in COVID-19. Heart, 106(15), 1132-1141. retrieved from https://doi.org/10.1136/heartjnl-2020-317056

27. Klok, FA., Kruip, MJHA., \& van der Meer, NJM. (2020). Incidence of thrombotic complications in critically ill ICU patients with COVID19. Thromb Res , doi:10.1016/j.thromres.2020.04.013. [retrieved from Epub ahead of print: 10 Apr 2020].

28. Li, B., Yang, J., \& Zhao, F. (2020). Prevalence and impact of cardiovascular metabolic diseases on COVID-19 in China. Clin Res Cardiol.

29. Liu, K., Fang, YY., Deng, Y., Liu, W., Wang, MF., Ma, JP., Xiao, W., Wang, YN., Zhong, MH., Li, CH., Li, GC., \& Liu, HG. (2020). Clinical characteristics of novel coronavirus cases in tertiary hospitals in Hubei Province. Retrieved October 20, 2020, from retrieved from https://pubmed.ncbi.nlm.nih.gov/32044814/

30. Liu, Z., Xiao, X., Wei, X., Li, J., Yang, J., \& Tan, H. (2020). Composition and divergence of coronavirus spike proteins and host 
ACE2 receptors predict potential intermediate hosts of SARS-CoV-2. J Med Virol. 20200226.

31. Marmor, M.F., Kellner, U., Lai, T.Y., Melles, R.B., \& Mieler, W.F. (2016). American Academy of Ophthalmology Recommendations on Screening for Chloroquine and Hydroxychloroquine Retinopathy (2016 Revision) Ophthalmology. Jun 2016;123(6):1386-1394. doi: 10.1016/j.ophtha.2016.01.058. Epub 2016 Mar 16.

32. Mehta, P., McAuley, DF., \& Brown, M. (2020). COVID-19: consider cytokine storm syndromes and immunosuppression. Lancet2020;395:10334. 10.1016/S0140-6736(20)30628-0

33. Mesquita, C.T., Ker, W.D., \& Azevedo, J.C. (2020). Cardiologia Nuclear em 2020 - Perspectivas da Nova Diretriz da Sociedade Brasileira de Cardiologia. Arquivos Brasileiros De Cardiologia. doi:10.36660/abc.20190874

34. Retallack, H., Di Lullo, E., \& Arias, C. (2016). Zika virus cell tropism in the developing human brain and inhibition by azithromycin. Proc Natl Acad Sci U S A 2016;113:14408-13. 10.1073/pnas.1618029113

35. Rice, GI., Thomas, DA., Grant, PJ., Turner, AJ., \& Hooper, NM. (2004). Evaluation of angiotensin-converting enzyme (ACE), its homologue ACE2 and neprilysin in angiotensin peptide metabolism. Biochem J. 2004;383(Pt 1):45-51.

36. Sahraei, Z., Shabani, M., \& Shokouhi, S. (2020). Aminoquinolines against coronavirus disease 2019 (COVID-19): chloroquine or hydroxychloroquine. Int J Antimicrob Agents2020;105945.

37. Seewoodhary, J. \& Oozageer, R. (2020). Coronavirus and diabetes: an update. Practical Diabetes, 37(2), 41.

38. Shi, S., Qin, M., Shen, B., Cai, Y., Liu, T., \& Yang, F. (2020). Association of cardiac injury with mortality in hospitalized patients with COVID-19 in Wuhan, China. JAMA Cardiol. 2020 Mar 25. retrieved from https://www.scielo.br/scielo.php?script=sci_nlinks\&pid=S0066782X202000060080500011\&lng=en

39. Shi, Z. \& Fu, W. (2020). Diagnosis and treatment recommendation for novel coronavirus pneumonia related isolated distal deep vein thrombosis. Shanghai Medical Journal, 2020. Available: http://kns.cnki.net/kcms/detail/31.1366.R.20200225.1444.004.html

40. Simoens, S., Laekeman, G., \& Decramer, M. (2013). Preventing COPD exacerbations with macrolides: a review and budget impact analysis. Respir Med 2013;107:637-48. 10.1016/j.rmed.2012.12.019

41. Tang, N., Bai, H., \& Chen, X. (2020). Anticoagulant treatment is associated with decreased mortality in severe coronavirus disease 2019 
patients with coagulopathy. J Thromb Haemost 2020. doi:10.1111/jth.14817. [Epub ahead of print: 27 Mar 2020].

42. Vaduganathan, M., Vardeny, O., Michel, T., McMurray, JJV., Pfeffer, MA., \& Solomon, SD. (2020). Renin-Angiotensin-Aldosterone System inhibitors in patients with COVID-19. N Engl J Med. 202003 30. retrieved from https://www.scielo.br/scielo.php?script=sci_nlinks\&pid=S0066782X202000060080500025\&lng=en]

43. Wan, Y., Shang, J., Graham, R., Baric, R. S., \& Li, F. (2020). Receptor recognition by the novel coronavirus from Wuhan: an analysis based on decade-long structural studies of SARS coronavirus. Journal of virology, 94(7).

44. Wang, D., Hu, B., \& Hu, C. (2020). Clinical characteristics of 138 hospitalized patients with 2019 novel coronavirus-infected pneumonia in Wuhan, China. JAMA 2020;323:1061-9. 10.1001/jama.2020.1585.

45. Wang, D., Hu, B., \& Hu, C. (2020). Clinical characteristics of 138 hospitalized patients with 2019 novel coronavirus-infected pneumonia in Wuhan, China. J Am Med Assoc. 2020

46. Wang, YD., Zhang, SP., \& Wei, QZ. (2020). COVID-19 complicated with DIC: 2 cases report and literatures review. Zhonghua Xue Ye Xue Za Zhi 2020;41:E001. 10.3760/cma.j.issn.0253-2727.2020.0001

47. Warren-Gash, C., Hayward, AC., Hemingway, H. (2020). Influenza infection and risk of acute myocardial infarction in England and Wales: a caliber self-controlled case series study. J Infect Dis 2012;206:1652-9. 10.1093/infdis/jis597

48. White, NJ. (2007). Cardiotoxicity of antimalarial drugs. Lancet Infect Dis 2007;7:549-58. 10.1016/S1473-3099(07)70187-1

49. Wu, Z. \& McGoogan, J.M. (2020). Characteristics of and important lessons from the coronavirus disease 2019 (COVID-19) outbreak in China: summary of a report of 72314 cases from the Chinese center for disease Control and prevention. J Am Med Assoc. 2020

50. Xiong, T.Y., Redwood, S., Prendergast, B., \& Chen, M. (2020). Coronaviruses and the cardiovascular system: acute and long-term implications. Eur Heart J. 2020

51. Xiong, TY., Redwood, S., Prendergast, B., \& Chen, M. (2020). Coronaviruses and the cardiovascular system: acute and long-term implications. Eur Heart J. 2020 Mar 18; retrieved from https://www.scielo.br/scielo.php?script=sci_nlinks\&pid=S0066782X202000060080500013\&lng=en

52. Xu, X., Chen, P., Wang, J., Feng, J., Zhou, H., \& Li, X. (2020). Evolution of the novel coronavirus from the ongoing Wuhan outbreak and modeling of its spike protein for risk of human transmission. Sci 
China Life Sci. 2020;63(3):457-60. [retrieved from https://www.scielo.br/scielo.php?script=sci_nlinks\&pid=S0066782X202000060080500022\&lng=en ]

53. Xu, X., Han, M., \& Li, T. (2020). Effective treatment of severe COVID-19 patients with tocilizumab. ChinaXiv 2020:20200300026.

54. Yamamoto, JM., Donovan, LE., Feig, DS., \& Berger, H. (2020). Urgent Update - Temporary Alternative Screening Strategy for Gestational Diabetes Screening during the COVID-19 Pandemic, 2020. Available from URl: retrieved from https://www.sogc.org/en/content/featured-news/GestationalDiabetesScreening-During-COVID-19-Pandemic.aspx

55. Yang, J. K., Lin, S.S., Ji, X.J., \& Guo, L.M. (2010). Binding of SARS coronavirus to its receptor damages islets and causes acute diabetes. Acta diabetologica, 47(3), 193-199.

56. Yao, X., Ye, F., Zhang, M., Cui, C., Huang, B., \& Niu, P. (2020). In Vitro Antiviral Activity and Projection of Optimized Dosing Design of Hydroxychloroquine for the Treatment of Severe Acute Respiratory Syndrome Coronavirus 2 (SARS-CoV-2) Clin Infect Dis. 2020 Mar 9 doi: 10.1093/cid/ciaa237. pii: ciaa237

57. Zhou, F., Yu, T., \& Du, R. (2020). Clinical course and risk factors for mortality of adult inpatients with COVID-19 in Wuhan, China: a retrospective cohort study. Lancet2020;395:1054-62. 10.1016/S01406736(20)30566-3

58. Zhou, F., Yu, T., \& Du, R. (2020). Clinical course and risk factors for mortality of adult inpatients with COVID-19 in Wuhan, China: a retrospective cohort study. Lancet. 2020

59. Zochios, V., Brodie, D., \& Charlesworth, M. (2020). Delivering extracorporeal membrane oxygenation for patients with COVID19: What, who, when and how ? Anaesthesia 2020. retrieved from https://doi.org/ 10.1111/anae.15099. 